\title{
The Effect of Manual Physical Therapy on Neck Disability Index in Myofascial Pain Syndromes: A Systematic Review
}

\author{
Chan-Myeong Kim
}

Korea Safety Health Environment Foundation, Work Environment Health Center

Received: August 17, 2020 / Revised: August 24, 2020 / Accepted: September 15, 2020

(c) 2020 J Korean Soc Phys Med

\begin{abstract}
| Abstract |
PURPOSE: The main purpose of this meta-analysis study was to identify the degree-of-effect size and the variables for the effects of manual physical therapy on myofascial pain syndrome.
\end{abstract}

METHODS: This study collected six studies published between 2015.01.01 and 2019.12.31. The analysis result verified nine effect size data. The random-effect model was chosen because of the heterogeneity of the data.

RESULTS: First, the full case showed the largest mean effect size of $2.297(p<.001)$. Second, the size of the effect based on the fascial distortion model (FDM) intervention showed an effect size of $4.654(\mathrm{p}<.001)$. Third, the number of participants showed a 15 or less effect size of 2.612 ( $p>$ .058). The number of treatments showed a 10 less effect size of $2.844(\mathrm{p}>$.129). The publication type showed a thesis effect size of $3.095(\mathrm{p}<.002)$.

CONCLUSION: Manual physical therapy has a great

Corresponding Author : Chan-Myeong Kim ptkcm@naver.com, https://orcid.org/0000-0001-7864-9561 This is an Open Access article distributed under the terms of the Creative Commons Attribution Non-Commercial License (http://creativecommons.org/licenses/by-nc/3.0) which permits unrestricted non-commercial use, distribution, and reproduction in any medium, provided the original work is properly cited. effect on myofascial pain syndrome in the neck and shoulders, and that the effects differ according to the methods of intervention.

Key Words: Myofascial pain syndromes, Manual therapy, Meta-analysis

\section{Introduction}

The increased use of computers has led to a rapid increase in posture alignment-related issues. Such posture alignment issues increase the forward head posture or rounded shoulder posture [1]. The forward head posture is caused mainly by an increase in sedentary lifestyle and the use of computers. Such posture is related to neck and shoulder pain and may cause a muscle imbalance [2]. In addition, comparative research on patients with chronic neck pain and normal people reported that patients with chronic neck pain show decreased kinesthesia [3]. Such forward head posture often causes myofascial pain syndrome (MPS) on the neck, and it is a common pain syndrome that anyone may experience once in a lifetime. Potential trigger points exist in $54 \%$ and $45 \%$ of adult women and adult men without symptoms, respectively, causing musculoskeletal pain [4]. This pain is caused by repeated microtrauma of the muscle and may be caused by excessive muscle shortening, excessive muscle 
extension by improper posture, excessive use of muscle, or desk and chair that do not fit the body [5]. Other than those, mental stress, nutritional imbalance, metabolic and endocrine imbalance, and infection have been pointed out as factors causing MPS [6]. Many patients have difficulty in treatment because the symptoms of this syndrome are often mistaken for the symptoms of other diseases [7]. The purpose of the treatment is to reduce pain and relieve the tension to damaged muscle [8]. MPS is an important disease that needs to be treated by physical therapists specialized in rehabilitation. Moreover, the treatment should be given by focusing on systematic and scientific evidence.

Meta-analysis is an evidence-based analysis method, and its goal is to analyze numerous individual studies done in the same themes to integrate the researches [9]. The strengths of meta-analyses are as follows. First, meta-analysis can make an organized and precise conclusion in integrating a series of research results. Second, meta-analysis can control the numerous flaws and biases in previous research. Third, meta-analysis can evaluate a population. Fourth, meta-analysis can identify the relationships among the research method, research target, scope, condition, and experiment period. On the other hand, meta-analysis has a weakness of focusing on results and ignoring the intervention of the moderating variables or interactions. As a result, meta-analysis may simplify the research result excessively [10]. Nevertheless, meta-analysis can enhance the statistical power by integrating the samples of individual research and testing the hypothesis with larger samples. Furthermore, meta-analysis can find the cause when there are conflicting research results. As a result, meta-analysis is a research method used widely in academia, such as medical science, public health, nursing, and pedagogy [11].

The NDI used in this study is a measuring tool with high reliability (ICC $=.59-.89$ ) in measuring neck pain [12]. In response, this study used meta-analysis to examine the effects of manual therapy methods and their influence on NDI evaluation in research for MPS on the neck and shoulder and identify the related variables.

\section{Methods}

The target of this study was the MPS on the neck and shoulder. This study used manual therapy as the intervention method and used different intervention methods from the targeted intervention for the comparative group. The study result was set to the neck disability index, and the study design type was set to the pre/post design experiment.

This study examined the following core question: 'What is the related variable for physical therapy on MPS?' Detailed selection standards were as follows: research targeting patients diagnosed with MPS on neck and shoulder, research suggesting an intervention of physical therapy, research suggesting statistical figures, experimental research conducted in a pre / post design, and research conducted within five years from January 1, 2015 to December 31, 2019. The following studies were excluded: research applying diagnosis, drug, injection, acupuncture, or surgery on MPS; research not conducted based on a pre/post design method; research without statistical figures; research without full text or research that was not reported in Korean or English.

The entire process of researching and selecting literature was conducted based on PRISMA systematic review flow chart [13]. This study used four databases, including the Korean Citation Index (KCI), Research Information Sharing Service (RISS), National Assembly Library, and DBpia. One hundred eighty-three papers were searched under the search keyword of 'myofascial pain'. The current researcher and experts specialized in physical therapy selected the materials. Mendeley and Excel were used to manage the selected studies. The materials were selected by checking the papers under the title and abstract and reviewing the original text in detail based on the selection and exclusion standards (Fig. 1.). 


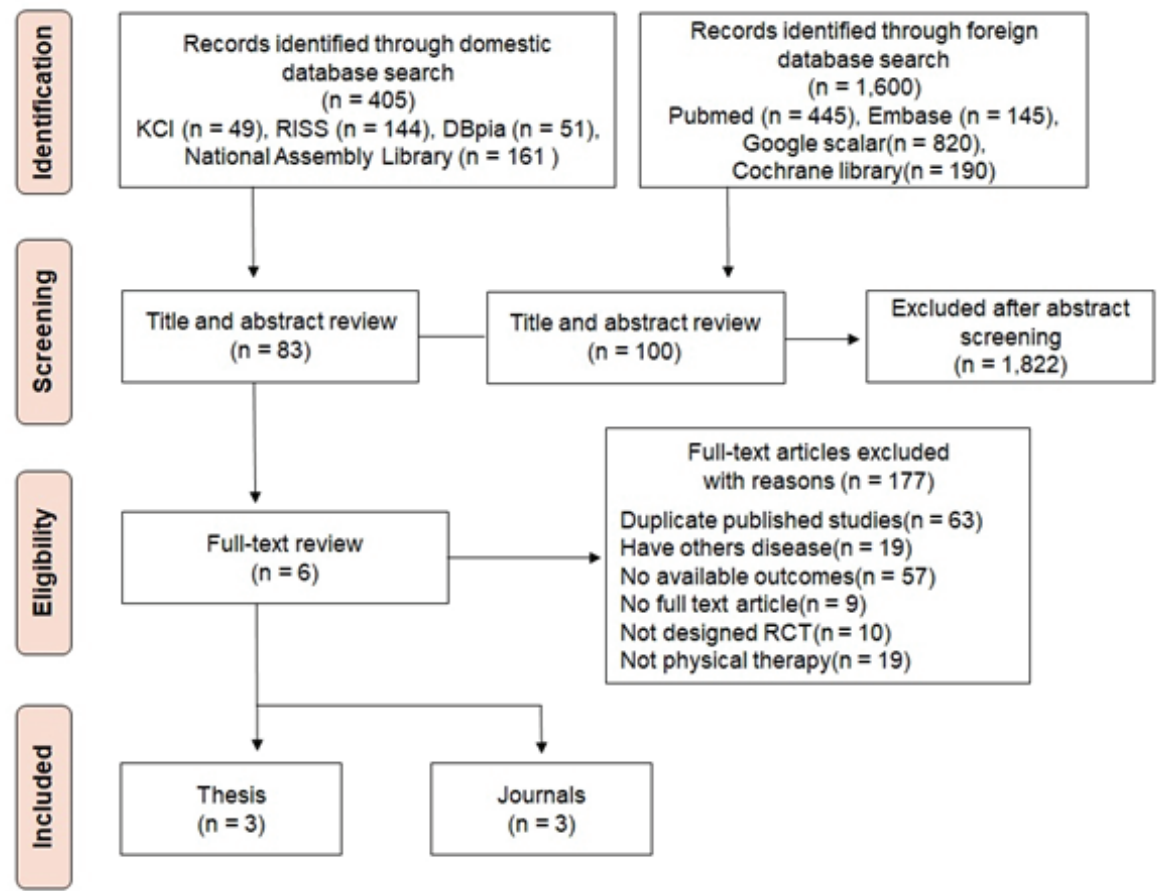

PRISMA: Preferred reporting items for systematic reviews and meta-analysis.

Fig. 1. "PRISMA" flow chart.

The Risk of Bias Verification Tool developed by the Cochrane Library and Review Manager 5.3 Program was used to evaluate the risk of bias on the 15 finally selected studies (Fig. 2.).

This study used a single-subject (pre-post test) metaanalysis method. Studies with a pre/ post mean and standard deviation for each experiment were selected. The collected materials were entered in Excel in the following order to make a coding sheet: researcher name, therapy method, intervention method, evaluation method, number of participants, therapeutic period, publication year, and thesis title. The coding data was used in the analysis by converting the data into csv file format to use in the R program. The dependent variables were evaluated by combining the evaluations showing positive (Positive number) and negative (Negative number) results. When the VAS showed a negative result, the pre-mean and standard deviation were replaced with the post-mean and standard deviation to suggest the all-sizes effect of a positive number to solve problems and derive comprehensive results.

R Program package used 'meta' and 'metafor', and each research's different intervention type and risk of bias were large. Therefore, this study calculated the size of the effect by applying a random-effect model. In addition, depending on the type of variables, this study analyzed the moderating effect using Meta-ANOVA on the categorical variables and Meta-Regression on the continuous variables. The effect size was interpreted as the standardized mean difference, and the method of interpreting the modified effect size was the same as Cohen's d interpretation [14].

Heterogeneity refers to the degree of the effect size distribution in each research and the degree of inconsistency. Therefore, the goal of meta-analysis was to understand the heterogeneity of effect size, the whole pattern instead of 

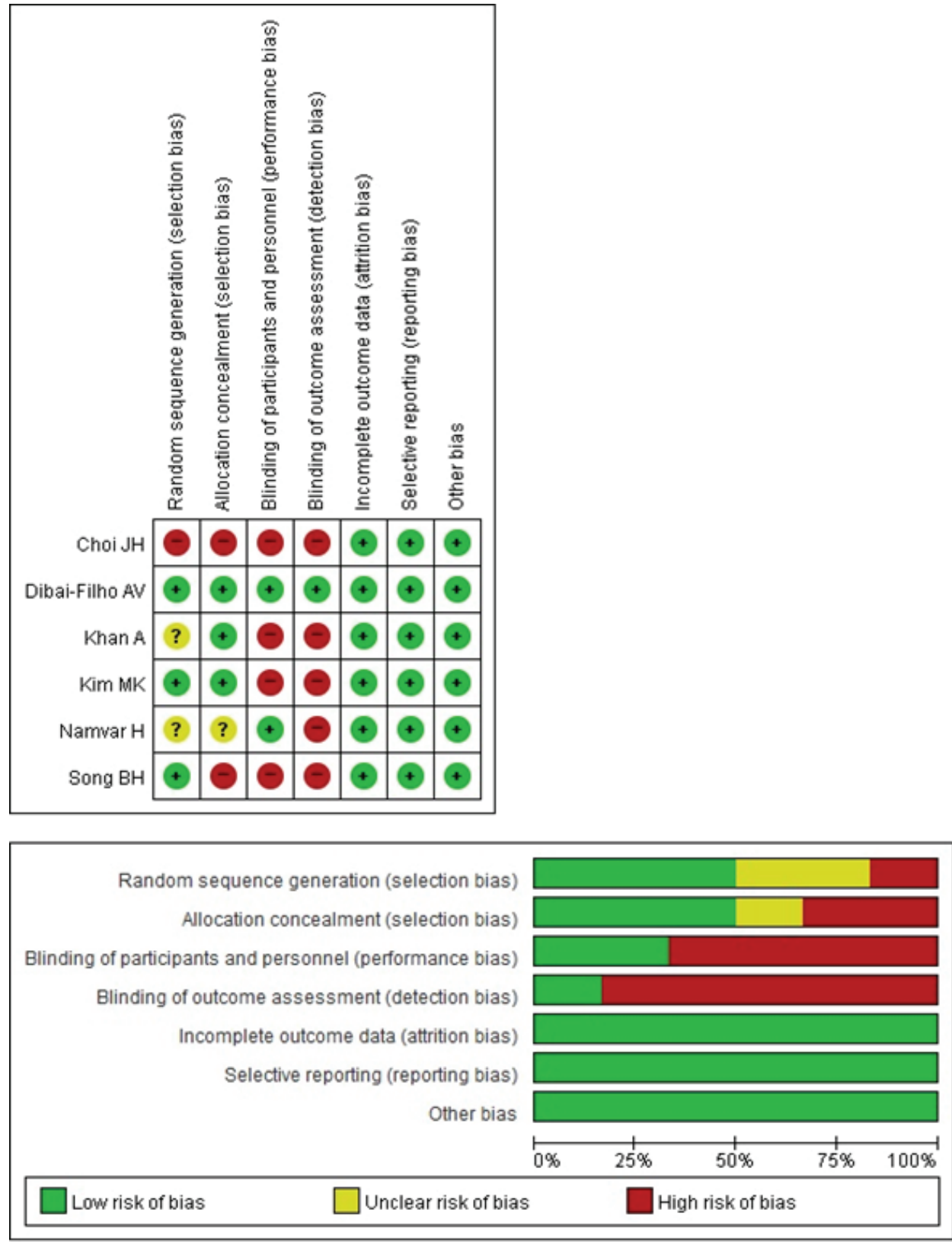

Study of risk of bias results.

Fig. 2. Risk of bias.

subtracting the mean of effect size [15]. Q and df were used to test the homogeneity in the effect size between the studies. The rate of heterogeneity is indicated in $\mathrm{I}^{2}$. The $\mathrm{I}^{2}$ statistics represent the rate of variance between the actual studies for the whole variance and represent the degree of heterogeneity in effect sizes deducted from individual researches15). $\mathrm{I}^{2}$ represents the actual variance (Between studies) for the entire variance (Total observed 


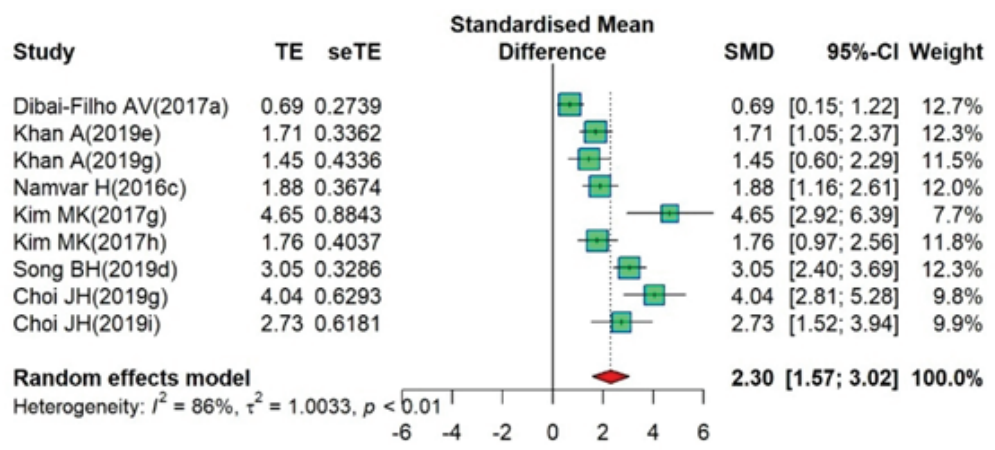

Effect size results for individual cases.

Fig. 3. Overall forest plot.

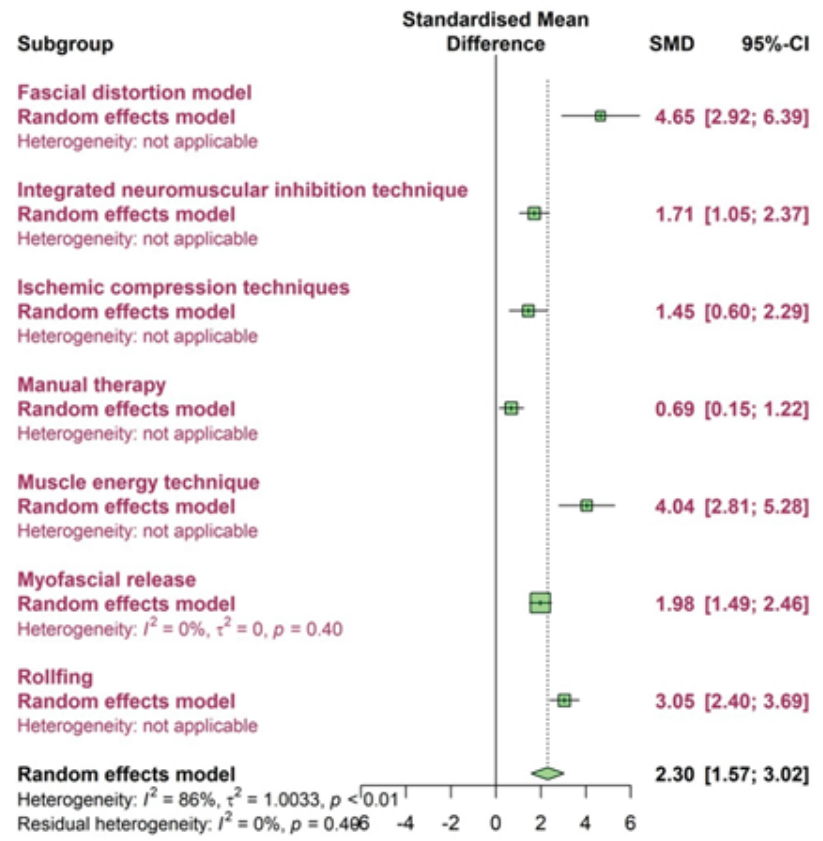

Effect size results by the intervention method.

Fig. 4. Intervention forest plot.

variance). In general, $\mathrm{I}^{2}$ of $25 \%$ is interpreted as low heterogeneity, $\mathrm{I}^{2}$ of $50 \%$ as moderate heterogeneity, and $\mathrm{I}^{2}$ over $75 \%$ as very high heterogeneity [16].

\section{Results}

The total effect size was significant $(p<.001)$ with a large effect size. The heterogeneity was high with $\mathrm{I}^{2}$ of $85.8 \%$ over $75 \%$ (Fig. 3.). In an analysis of the intervention types, the fascial distortion model was highest with $(\mathrm{ES}=4.654)$, and all were statistically significant at the $95 \%$ confidence interval $(p<.001)$ (Fig. 4.).

Regarding the number of cases, the effect size for the number of patients was high in the number of patients 
Table 1. Treatment ES According to the Control Variable

\begin{tabular}{|c|c|c|c|c|c|c|c|}
\hline \multirow{2}{*}{ Control Variable } & \multirow{2}{*}{$\mathrm{N}$} & \multirow{2}{*}{$\mathrm{k}$} & \multirow{2}{*}{ ES (g) } & \multicolumn{2}{|c|}{$95 \% \mathrm{CI}$} & \multirow{2}{*}{ Q (df) } & \multirow{2}{*}{$\mathrm{p}$} \\
\hline & & & & LL & UL & & \\
\hline \multirow{2}{*}{ No. of Participants } & $>15$ & 2 & 1.261 & .087 & 2.435 & \multirow{2}{*}{$3.59(1)$} & \multirow{2}{*}{$>.058$} \\
\hline & $\leq 15$ & 7 & 2.612 & 1.852 & 3.373 & & \\
\hline \multirow{2}{*}{ No. of Treatments } & $<10$ & 5 & 2.844 & 1.846 & 3.843 & \multirow{2}{*}{$2.31(1)$} & \multirow{2}{*}{$>.129$} \\
\hline & $\geq 10$ & 4 & 1.718 & .664 & 2.772 & & \\
\hline \multirow{2}{*}{ Publication Type } & Journal & 4 & 1.401 & .807 & 1.994 & \multirow{2}{*}{$9.48(1)$} & \multirow{2}{*}{$<.002$} \\
\hline & Thesis & 5 & 3.095 & 2.194 & 3.995 & & \\
\hline
\end{tabular}

k: Number of effect size; ES: Effect size; 95\% CI: 95\% Confidence interval

LL: Lower limit; UL: Upper limit

Table 2. Calibration Results for 11 Cases Through "Trim and Fill".

\begin{tabular}{cccccccc}
\hline \multirow{2}{*}{ Publication Bias } & \multirow{2}{*}{$\mathrm{k}$} & $\mathrm{ES}(\mathrm{g})$ & \multicolumn{3}{c}{$95 \% \mathrm{CI}$} & \multirow{2}{*}{$\mathrm{Q}(\mathrm{df})$} & $\mathrm{p}$ \\
\cline { 5 - 6 } & & & $\mathrm{LL}$ & $\mathrm{UL}$ & & \\
\hline Trim and Fill & 11 & 1.833 & 1.071 & 2.596 & $82.32(10)$ & $<.001$ \\
\hline
\end{tabular}

k: Number of effect size; ES: Effect size; 95\% CI: 95\% Confidence interval LL: Lower limit; UL: Upper limit

below 15, but the difference was not significant $(\mathrm{p}>.058)$. With the number of cases and effect sizes for the therapeutic period, the figures were high in less than 10 days, but the difference was not significant $(\mathrm{p}>.129)$. Regarding the number of cases and the effect size for the publication type, the figures were high in the thesis showing statistical significance $(\mathrm{p}<.002)$, (Table 1). To analyze the publication error, this study used 'Trim and Fill' and corrected the asymmetry to symmetry [17]. As a result, two cases were added, and the effect size corrected with 11 cases was $(\mathrm{ES}=1.833)$, showing statistical significance $(\mathrm{p}<.001)$ (Table 2) (Fig. 5.).

\section{Discussion}

This study collected studies that applied manual therapies on MPS of the neck and shoulder and examined the effect on the NDI results and more effective therapeutic methods based on a systematic and integrative meta-analysis.
In this study, the total effect size was ( $\mathrm{ES}=2.297$ ), and the effect size was $(E S=1.833)$ after correcting for the publication bias. This showed that manual therapy largely improved the result of NDI toward MPS on the neck and shoulder. In particular, the effect size of the FDM was large $(\mathrm{ES}=4.654)$. According to Kim [18], fascial therapy had a positive effect because of GTO and the function of the muscle spindle. Moreover, the FDM was the fastest and the most effective therapeutic method for controlling the function and pain of MPS. Manual therapy transmits pressure to the muscle to induce intermittent muscular contraction in certain intervals, increase the blood flow rate in the muscle with lower blood flow, supply oxygen and nutrition to lower tension, relieve pain, control sympathetic nerve, and increase movable range [19].

In the moderating variable, the number of cases and effect sizes were high for 15 patients, but the difference was not significant. Therefore, more research on more patients will be needed to make up for the reliability. In 

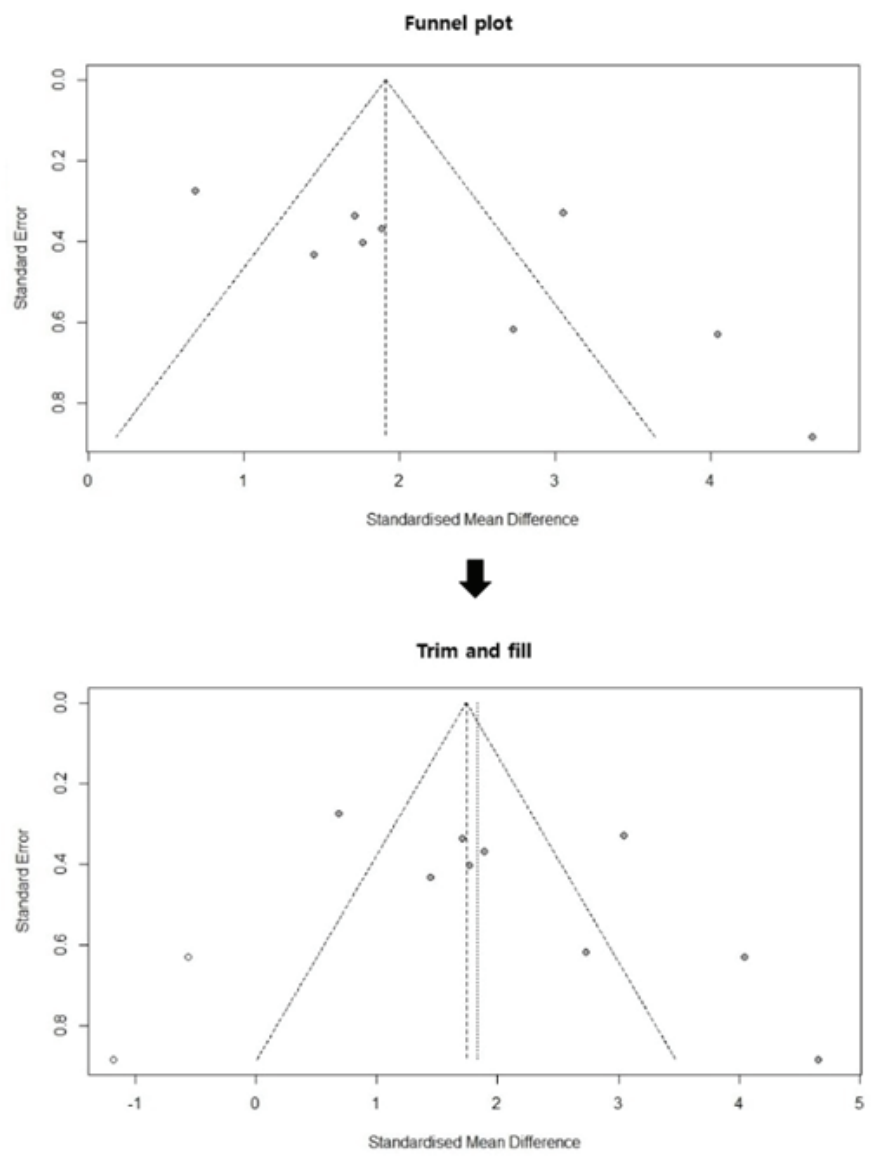

Calibration results for 11 cases through "Trim and Fill".

Fig. 5. Funnel plot and trim and fill.

addition, the short-term exercise intervention relieved pain and enhanced the body function [20]. On the other hand, more research will be needed to examine the therapeutic effect from a more long-term perspective. In the publication type, the number of cases and effect sizes were high for the thesis. This requires an interpretation considering that this study included both masters and doctorate theses in the thesis group.

To verify the appropriateness of the meta-analysis result, this study corrected the asymmetry to symmetry. By adding the two cases assumed not to be reported due to publication bias, the adjusted effect size was ( $E S=1.833)$, and the $95 \%$ confidence interval was (1.071 2.596), showing a significant result. This study has some limitations. Although the literature was selected by experts and result corrected by utilizing Trim and Fill was suggested to minimize the publication bias, the limit of meta-analysis for publication bias could not be solved completely. Therefore, future research should extend the scope of study and conduct a meta-analysis, including studies of various cases.

\section{Conclusion}

Manual therapy has a huge effect on the NDI result for MPS on the neck and shoulder and is influenced by the number of patients, therapeutic period, number of 
therapies, and publication type. Overall, the study results suggest material for the more effective application of manual therapy systematically and objectively by metaanalysis.

\section{References}

[1] Kang JH, Park RY, Lee SJ, et al. The effect of the forward head posture on postural balance in long time computer based worker. Ann Rehabil Med 2012;36:98.

[2] Silva AG, Punt TD, Sharples P, et al. Head posture and neck pain of chronic nontraumatic origin: a comparison between patients and pain-free persons. Arch Rehabil Res Clin Transl 2009;90:669-74.

[3] Revel M, Andre-Deshays C, Minguet M. Cervicocephalic kinesthetic sensibility in patients with cervical pain. Arch Rehabil Res Clin Transl 1991;72:288-91.

[4] Simons DG, Travell J, Simons LS. Myofascial pain and dysfunction: the trigger point manual: volume 11999.

[5] Lee JH, MS KOH, MS YHP. Comparing the effects of stability exercise, ESWT, and taping for patients with myofascial pain syndrome of upper trapezius. J Kor Phys Ther 2012;24:82-9.

[6] Gerwin RD. A review of myofascial pain and fibromyalgiafactors that promote their persistence. Acupunct Med 2005;23:121-34.

[7] Kang EK: Exercise and Physical Therapy for the Musculoskeletal Pain. Medical Postgraduates 2005;33: 149-53.

[8] Hsueh TC, Cheng PT, Kuan TS, et al. THE IMMEDIATE EFFECTIVENESS OF ELECTRICAL NERVE STIMULATION AND ELECTRICAL MUSCLE STIMULATION ON MYOFASCIAL TRIGGER POINTS1. Am J Phys Med Rehabil 1997;76:471-6.

[9] Glass G V. Primary, secondary, and meta-analysis of research. Educational Researcher 1976;5:3-8.

[10] Jackson GB. Methods for integrative reviews. Rev Educ Res 1980;50:438-60.

[11] Lee HJ, Oh HH, Choi KH. Meta analysis on the effect of reading development program on reading comprehension. Journal of the Korean Data and Information Science Society 2012;23:447-55

[12] MacDermid JC, Walton DM, Avery S, Blanchard A, Etruw E, Mcalpine C, et al. Measurement properties of the neck disability index: a systematic review. J Orthop Sports Phys Ther 2009;39:400-17.

[13] Moher D, Liberati A, Tetzlaff J, et al. Preferred reporting items for systematic reviews and meta-analyses: the PRISMA statement. PLoS Med 2009;6:e1000097.

[14] Cohn J. Statistical power analysis for the behavioral sciences. Lawrence Earlbam Associates, Hillsdale, NJ 1988.

[15] Borenstein M, Hedges L V, Higgins JPT, et al. Introduction to meta-analysis. John Wiley \& Sons; 2011.

[16] Higgins JP, Green S. Cochrane Handbook for Systematic Reviews of Interventions Version 5.1. 0 [document on the Internet]. The Cochrane Collaboration Available from: URL: Http://Www Cochrane-Handbook Org 2011.

[17] Duval S, Tweedie R. Trim and fill: a simple funnelplot-based method of testing and adjusting for publication bias in meta-analysis. Biometrics 2000;56:455-63.

[18] Kim MK, Lee WJ. Effect of fascial distortion model on the pain and movement of neck patient. J Kor Phys Ther 2019;31:24-30.

[19] Fryer G. Muscle energy technique: An evidence-informed approach. Int J Osteopath Med 2011;14:3-9.

[20] Li Y, Su Y, Chen S, Zhang Y, et al. The effects of resistance exercise in patients with knee osteoarthritis: a systematic review and meta-analysis. Clin Rehabil 2016;30:947-59. 